Note on Comet c, 1874, and Mr. Birmingham's Variable Star in Monoceros.

Since my communications of the $13^{\text {th }}$ ultimo was forwarded to you I have discovered that the comparison star of the $81 / 2 \mathrm{mag}$. taken from the Melbourne list is not the one actually employed in the differential observations of the Comet on the $24^{\text {th }}$ August. The AR. and N. P. D. of the Melbourne star are about $37^{\prime}$ and $2^{\prime}$ too small respectively. The accurate position of the comparison star and also that of the star employed on the $7^{\text {th }}$ September, neither of which has been observed at Melbourne, will be determined differentially at an early opportunity. For the reason just stated the position of the Comet as forwarded for the $24^{\text {th }}$ August will be found to be considerably in error.

The star described by $\mathrm{Mr}$. Birmingham in $\mathrm{Nr}$. 2028 of the Astr. Nachr was observed here with the $4^{1 / 2}$ inch equatorial on the evenings of the $8^{\text {th }}$ and $9^{\text {th }}$ instant. It was of a red-orange colour, and considerably brighter thare B. A. C. 2470. Its image in the telescope was, however, more disturbed and worse defined than that of B. A. C. 2470. The B. A. Catalogue gives 6.0 as the magnitude of the latter, but the Radcliffe Observations for 1858 make it of the $6.7 \mathrm{mag}$. Adopting the latter as the preferable estimate $I$ consider the supposed variable to be about 6.3 mag. Mr. Birmingham assigns 7.3 as the magnitude on the $14^{\text {th }}$ February last (see Astronomical Register for April 1875). If our estimates be at all comparable the star is now about a whole magnitude greater than it was in February. Its position as approximately determined by circle comparisons with B. A. C. 2470 is $\mathrm{AR}=7^{\mathrm{h}} 23^{\mathrm{m}} 5$, N.P.D. $=100^{\circ} 4^{\prime}$.

Private Observatory Windsor, N.-S.-Wales. 1875, June 10.

John Tebbutt.

\title{
Elemente und Ephemeride des Planeten (148).
}

Ich beehre mich, Ihnen die folgenden Elemente nebst Ephemeride des von Herrn P. Henry entdeckten Planeten mitzutheilen, welche ich ans den Beobachtungen Paris Ang. 7, und Berlin Aug. 14 und 23 abgeleitet habe: Epoche 1875, August 14.5087.

$\mathrm{M}=311^{\circ} 23^{\prime} \quad 3^{\prime \prime} 4$

$\omega=254 \quad 1859.6$

$\left.\delta=\begin{array}{rrr}145 & 18 & 4.1\end{array}\right\}$ mittl. Aequ. 1875.0

$\mathrm{i}=25 \begin{array}{lll}57 & 19.2\end{array}$

$\varphi=114153.3$

$\log \mathrm{a}=0.448222$

$\mu=754^{\prime \prime} 525$

Mittlerer Ort 1875.0.

$1875,12^{\mathrm{h}}$ Berlin $\quad \alpha \quad \delta \quad \log \triangle \quad \log \mathrm{r}$

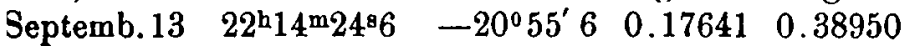

1347.1

$\begin{array}{llll}15 & 13 & 10.6 & 22.6\end{array}$

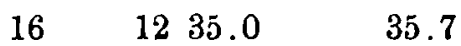

$\begin{array}{llllllll}17 & 12 & 0.5 & -21 & 48.5 & 0.18114 & 0.38819\end{array}$

$\begin{array}{lllll}18 & 11 & 27.1 & -22 & 1.0\end{array}$

$\begin{array}{llll}19 & 10 & 54.9 & 13.3\end{array}$

$\begin{array}{llll}20 & 1023.8 & 25.3\end{array}$

$\begin{array}{llllll}21 & 9 & 54.0 & 36.9 & 0.18688 & 0.38688\end{array}$

$22 \quad 925.4 \quad 48.3$

$\begin{array}{lllll}23 & 8 & 58.2 & -22 & 59.4\end{array}$ $1875,12^{\mathrm{h}}$ Berlin $\quad \alpha \quad \delta \quad \log \Delta \quad \log \mathrm{r}$ Septemb. $24 \quad 22^{\mathrm{h}} 8^{\mathrm{m}} 32^{\mathrm{B}} 3 \quad \mathbf{2 3}^{0} 10^{\prime} 2$

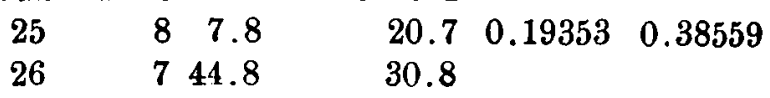

$\begin{array}{lll}744.8 & 30.8\end{array}$

$\begin{array}{lll}27 & 7 \cdot 23.3 & 40.7\end{array}$

$\begin{array}{llll}28 & 7 & 3.3 & 50.3\end{array}$

$\begin{array}{lllllll}29 & 6 & 44.8 & -23 & 59.6 & 0.20096 & 0.38431\end{array}$

$\begin{array}{lllll}30 & 6 & 27.9 & -24 & 8.5\end{array}$

$\begin{array}{lllll}\text { October } & 1 & 6 & 12.7 & 17.2\end{array}$

$2 \quad 559.1 \quad 25.5$

$\begin{array}{lllllll}3 & 5 & 47.1 & 33.6 & 0.20906 & 0.38304\end{array}$

$\begin{array}{llll}4 & 5 & 36.8 & 41.3\end{array}$

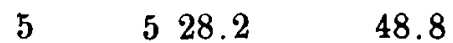

$\begin{array}{lllll}6 & 5 & 21.3 & -24 & 55.9\end{array}$

$\begin{array}{llllllll}7 & & 5 & 16.1 & -25 & 2.7 & 0.21770 & 0.38178\end{array}$

$\begin{array}{llll}8 & 5 & 12.7 & 9.2\end{array}$

$\begin{array}{llll}9 & 5 & 11.0 & 15.4\end{array}$

$10 \quad 511.0 \quad 21.3$

$\begin{array}{lllllll}11 & 5 & 12.8 & & 27.0 & 0.22677 & 0.38054\end{array}$

$\begin{array}{llll}12 & 5 & 16.3 & 32.3\end{array}$

$13 \quad 521.6 \quad 37.4$

$\begin{array}{llll}14 & 5 & 28.5 & 42.2\end{array}$

$\begin{array}{llllllll}15 & 22 & 5 & 37.2 & -25 & 46.7 & 0.23615 & 0.37931\end{array}$

Berlin. 1875, September $11 . \quad V$. Knorre.

Beobachtung des Planeten (148) auf der Leipziger Sternwarte. 175, September $2,1^{\mathrm{h}} 29^{\mathrm{m}} 6^{\mathrm{B}}$ mittlere Leipziger Zeit $\alpha$ app. $22^{\mathrm{h}} 23^{\mathrm{m}} 3^{\mathrm{s}} \mathrm{g} 2 \delta$ app. $-18^{\mathrm{O}} 19^{\prime} 46^{\prime \prime} 9$

C. Bruhns. 